\title{
Les municipalités à la conquête des réseaux sociaux. Mais pour quoi faire?
}

Ewa Krzatala-Jaworska

\section{(2) OpenEdition}

1 Journals

Édition électronique

URL : http://journals.openedition.org/communicationorganisation/4151

DOI : 10.4000/communicationorganisation.4151

ISSN : $1775-3546$

Éditeur

Presses universitaires de Bordeaux

Édition imprimée

Date de publication : 1 juin 2013

Pagination : 86-104

ISBN : 978-2-86781-883-7

ISSN : $1168-5549$

\section{Référence électronique}

Ewa Krzatala-Jaworska, «Les municipalités à la conquête des réseaux sociaux. Mais pour quoi faire? ", Communication et organisation [En ligne], 43 | 2013, mis en ligne le 01 juin 2015, consulté le 19 avril 2019. URL : http://journals.openedition.org/communicationorganisation/4151 ; DOI : 10.4000/ communicationorganisation. 4151 


\title{
Les municipalités à la conquête des réseaux sociaux Mais pour quoi faire?
}

\author{
Ewa Krzatola-Jaworska'
}

Les collectivités locales misent de plus en plus sur la communication numérique. Dans les années 1990, les municipalités françaises commencent à lancer leurs sites web ${ }^{2}$ qui, depuis, deviennent de plus en plus interactifs grâce à la possibilité de contacter les élus via e-mail, blog ou forum de discussion. Aujourd'hui, de nombreuses municipalités marquent leur présence sur les réseaux sociaux ou même investissent dans la création de réseaux sociaux locaux ${ }^{3}$ propres à leur territoire. Les collectivités locales semblent ainsi partager l'opinion selon laquelle les réseaux sociaux joueraient de nos jours un rôle important dans la communication politique.

Ce constat empirique est confirmé par la revue de la littérature spécifique, qui est très prolifique dans le domaine de marketing territorial et de sciences de l'information et de communication. Les recherches existantes permettent de constater que la présence des collectivités territoriales sur Facebook est une tendance générale en France, surtout au niveau des régions et des départements. L'enquête de Michel Barabel nous indique que « l'intégralité des territoires français ont des groupes sur Facebook et ce phénomène n'est, semble $\mathrm{t}$-il, pas marginal puisqu'il concerne également les départements ou les régions les moins connues ${ }^{4}$. Cette même tendance est également vraie pour les municipalités. Leur présence sur les réseaux sociaux est de plus perçue comme une étape du phénomène bien plus général d'amplification des moyens numériques de communication politique initié par l'apparition des réseaux télématiques dans les années 80 , alimenté par le développement d'Internet et dynamisé par l'avenant du « web participatif » appelé aussi « web 2.0. »5. Dans

1 Doctorante en science politique à l'Université Paris 1, CRPS-CESSP ; Ewa.Krzatala-Jaworska@univ-paris1.fr 2 G. LOISEAU, « La démocratie numérique municipale française : au-delà des parangons de vertu », Hermès, $\mathrm{n}^{\circ} 26-27,2000, \mathrm{p} .213$.

3 Cf. le cas d'Issy-les-Moulineaux qui a lancé son réseau social local iFolio http://www.i-folio.fr/.

4 M. BARABEL et al., " Les médias sociaux au service du marketing territorial : une approche exploratoire ", Management Eं Avenir, n 32, 2/2010, p. 245.

5 G. LOISEAU, « Les sites internet des municipalités, les blogs et les réseaux sociaux: vers une multiplication des modalités participatives dans l'espace public local? » in E. FOREY, C. GESLOT, Internet, machines à voter 
cette perspective, bien plus vaste, l'usage des réseaux sociaux par les collectivités locales devrait être interprété, comme le font Philippe Bouquillion et Isabelle Pailliart pour les Sites Internet Municipaux (SIM), comme étant une preuve de la banalisation de l'Internet dans les pratiques sociales et un élément de la stratégie de diversification des outils de communication ${ }^{6}$.

La popularité des réseaux sociaux parmi les collectivités territoriales se donne à voir également à travers l'émergence de ressources sur lesquelles les collectivités locales peuvent s'appuyer pour choisir la meilleure stratégie de conquête des réseaux sociaux. L'apparition de cabinets de conseils spécialisés, la naissance du nouveau métier de gestionnaire de communauté, des sites web qui offrent leurs astuces et guident les collectivités dans leurs actions sur les réseaux sociaux sont seulement quelques exemples de ce phénomène. Gérard Loiseau, qui étudie la communication politique numérique des municipalités françaises depuis les années 80, constate qu'actuellement en France 14 villes sur 43 utilisent les réseaux sociaux : 13 utilisent Facebook, 12 utilisent Twitter et 11 tous les deux ${ }^{7}$.

La revue de la littérature ainsi que les tendances observables sur la toile prouvent que la présence des collectivités locales sur les réseaux sociaux est un fait qui interroge à la fois les chercheurs des domaines divers et les acteurs des collectivités. Les questions les plus souvent posées concernent la promotion du territoire (marketing territorial), la multiplication de la communication institutionnelle (sciences de l'information et de communication) et les rapports entre les élus et les citoyens dans ce nouveau contexte communicationnel (sciences de l'information et de la communication/science politique). Les recherches existantes se focalisent donc sur les conséquences de l'usage des réseaux sociaux tout en restant relativement silencieuses sur les motifs de ce choix et les raisons pour lesquelles les collectivités locales consacrent du temps et de l'énergie à ces nouvelles arènes publiques, comme si l'usage des réseaux sociaux allait de soi. On sait que l'usage de ces dispositifs n'est pas celui rêvé des théoriciens de la démocratie participative, car l'information et le management politique dominent l'interaction et le dialogue. Mais on ne sait ni pourquoi les collectivités locales souhaitent être présentes sur les réseaux sociaux ni pourquoi une grande partie de projets municipaux sur les réseaux sociaux n'est pas suivie par les usagers.

Notre travail vise donc à déterminer les enjeux et les limites de l'usage des réseaux sociaux par les collectivités territoriales. Dans notre réflexion, nous allons faire le lien entre trois éléments qui font objet d'un débat actuel. Nous partons du constat, rappelé ci-dessus, de la présence des institutions politiques sur les réseaux sociaux afin de pouvoir étudier l'usage de ces derniers fait

et démocratie, L'Harmattan, Paris, 2011, p. 191-213.

6 P. BOUQUILLION, I. PAILLIART, Déploiement des Tic dans les territoires. Le rôle des collectivités, Presses

Universitaires de Grenoble, Grenoble, 2006, p. 14.

7 G. LOISEAU, art. cit., 2011, p. 197. 
par les collectivités locales et pour enfin arriver à déterminer si les réseaux sociaux constituent un outil de la communication publique. L'objectif de cette étude consiste donc à confronter la présence massive de collectivités territoriales sur les réseaux sociaux avec les usages effectifs de ces outils. En dépit des travaux mentionnés ci-dessus, cet objet de recherche reste toujours peu exploité contrairement au rôle des réseaux sociaux pour les mouvements sociaux $^{8}$ (ex. printemps arabe), pour les partis politiques ${ }^{9}$ (CooPol, Créateurs de possibles) ou bien pour la campagne électorale ${ }^{10}$ (ex. celle de Barack Obama de 2008). Notre étude du cas présenté plus loin peut donc d'autant plus être une contribution importante au débat en cours.

Pour répondre à la problématique signalée ci-dessus, nous nous sommes appuyée sur le travail de terrain effectué à Fontenay-sous-Bois en 2010 où nous avons étudié le cas des Etats-Généraux de la Ville Numérique, une concertation publique hors ligne et en ligne portant sur le rôle du numérique dans la ville. L'encadré 1 présente le projet en détail.

Pour étudier ce cas, nous avons combiné des méthodes quantitatives et qualitatives. L'étude de la communication numérique autour du projet municipal (exploitation des données statistiques du site web dédié au projet www.fontenaynumerique.net. et analyse du contenu de sa page Facebook) a été complétée par des entretiens semi-directifs avec le webmestre du site qui est aussi l'administrateur du compte Facebook/Twitter du projet ainsi qu'avec les autres gestionnaires de communauté du compte Facebook. Le contexte politique et administratif du projet a été retracé grâce aux archives de la mairie.

Notre texte vise à déterminer dans quelle mesure les réseaux sociaux peuvent devenir un outil de communication politique autour d'un projet municipal. Dans un premier temps, nous présenterons les enjeux politiques et communicationnels de la présence des municipalités sur les réseaux sociaux et nous finirons notre réflexion en démontrant les limites de cet usage des réseaux sociaux.

8 Voir par exemple : D. FARIS, Dissent and Revolution in a Digital Age: Social Media, Blogging and Activism in Egypt, Londres, I.B.Tauris, 2012.

9 Voir par exemple : F. GREFFET, Continuerlalutte.com. Les partis politiques sur le web, Paris, Presses de Sciences Po « Académique », 2011.

10 Voir par exemple : D. TALBOT, « How Obama Really Did It », Technology Review, 111, 5, p. 78-83, 2008. 


\section{Encadré 1}

Généraux de la Ville Numérique est une concertation publique organisée du 4 avril 2009 au 5 mai 2010 par la mairie de Fontenay-sous-Bois autour du rôle du numérique dans quatre domaines : la démocratie locale, la solidarité, l'éducation et le développement durable. La démarche se composait de plusieurs étapes : un séminaire d'information et de discussion pour les élus a été suivi de 16 réunions publiques (4 pour chaque sujet abordé) et un colloque intitulé « Internet pour tous ", qui a rassemblé des spécialistes de ces sujets et des représentants d'autres villes connaissant des projets de numérisation réussis, a été organisé.

Le projet s'inscrit dans cette démarche participative et constitue un volet des assises de la ville, donc en opérant une grande consultation des citoyens. L'aspect participatif des États Généraux est mis en avant de manière très prégnante dans le discours officiel lié à ce projet. Ainsi, il est directement attaché à 140 engagements municipaux ; on peut par exemple lire : «La municipalité attachée au développement de la démocratie participative a souhaité associer dans la réflexion les citoyens, les élus et les agents publics ${ }^{11}$.

La consultation des citoyens dans le cadre d'États Généraux de la Ville Numérique a été organisée de deux façons : en face-à-face, à travers les 16 réunions organisées en semaine entre $18 \mathrm{~h} 30$ et 20h30, et à distance, via le site web dédié au projet dont l'URL est le suivant: www.fontenaynumerique. net. Cette dernière facette des États Généraux permet d'analyser l'apport des dispositifs du web 2.0 à la promotion d'un projet politique.

La documentation sur le projet ainsi que les interviews avec les porteurs du projet permettent d'en distinguer plusieurs objectifs. Tout d'abord, les États Généraux de la Ville Numérique font partie de la communication politique des élus de la Ville par leur attachement direct aux 140 engagements du Conseil Municipal. Dans le cadre du projet les élus rappellent leurs engagements liés au numérique et confirment leur volonté de tenir leurs promesses. Ensuite, le projet a pour but d'engager une discussion publique sur le rôle du numérique dans la vie de tous les jours. D’un côté, il était donc conçu comme un élément de la démocratie participative dans la ville, de l'autre, il doit faire travailler ensemble les acteurs différents engagés dans cette thématique afin de les faire discuter et qu'ils nouent des relations entre eux. L'important, pour les porteurs du projet, est que les outils de communication numérique doivent faciliter la discussion et réunir les acteurs intéressés par cette problématique.

$11 \mathrm{Cf}$. le projet des États Généraux, archives de la DSIT de Fontenay-sous-Bois. 


\section{La communication politique à la conquête des réseaux sociaux.}

Suivant une tendance générale à l'élargissement de l'ampleur des réseaux sociaux visible aussi bien en France qu'à l'étranger, les porteurs du projet États Généraux de la Ville Numérique ont décidé d'être présents sur les réseaux sociaux. Leur choix s'est porté sur deux réseaux très populaires : Facebook et Twitter. Dans la partie qui suit, nous allons démontrer les enjeux sociopolitiques et communicationnels ayant influencé cette stratégie.

\section{Les enjeux sociopolitiques.}

Les enjeux d'utilisation des réseaux sociaux pour la promotion d'un projet municipal seront tout d'abord présentés sous l'angle sociopolitique. Dans quelle mesure l'usage des réseaux sociaux peut-il contribuer à l'image de la ville? Peut-il aussi faciliter la communication politique permettant à la fois d'atteindre un public large et de viser une population d'habitude peu intéressée par le politique?

Selon certains auteurs, le réflexe d'utiliser Facebook vient du fait que quelques-unes de ses caractéristiques faciliteraient la communication politique $^{12}$. Premièrement, il permettrait d'atteindre un «vrai » espace public, car il n'existe aucune autre communauté qui réunisse autant de gens malgré les distances géographiques et autres. Deuxièmement, ce réseau combinerait les meilleures qualités du bulletin municipal, des réunions publiques et des journaux. Contrairement aux réunions publiques, les sujets abordés sur Facebook ne seraient pas imposés par les autorités mais choisis par les internautes. En plus, le message serait communiqué à ceux qui le souhaitent. Finalement, grâce à Facebook les politiciens pourraient avoir une réaction de leurs électeurs sur leurs propos.

Le but principal des Etats Généraux de la Ville Numérique tel qu'il est mis en avant par les porteurs du projet, est de faire discuter des représentants des milieux et des groupes sociaux des enjeux liés aux TIC. Il s'agit en particulier de nouer des liens entre les associations, bailleurs sociaux, professionnels, enseignants et travailleurs sociaux. Le responsable du projet, chef de la Direction des Systèmes de l'Information et de la Télécommunication de la mairie souligne cet objectif dans un entretien:

"On a réussi à voir beaucoup de monde quand même et de solliciter la réflexion des uns et des autres. Souvent ils conduisent des projets liés d'une certaine façon au numérique, mais ces projets, même si très proches, sont déconnectés. Pour donner un exemple ce sont les enseignants et l'association des parents d'élèves, ou des travailleurs sociaux et les associations qui aident la population défavorisée. On a aussi réussi à nouer des relations avec des différentes agences de l'État ce qui nous permettra de mener notre politique dans l'avenir ».

12 M.WESTLING, «Expanding the Public Sphere:The Impact of Facebook on the Political Communication », Society, vol. 28, 2007, p. 835-860. 
Dans cette optique, les réseaux sociaux sont perçus comme un outil important de la mise en relation des individus et des groupes qui pourraient collaborer pour conduire leurs projets pro publico bono. D'une part, avant les réunions physiques à la mairie, ils pourraient se familiariser déjà avec d'autres personnes qui y participent en consultant leurs pages Facebook et en lisant leurs propos posté sur le wall du compte du projet. D'autre part, après les réunions physiques, le compte Facebook du projet doit se transformer en lieu de débat prolongé à distance et renforcer les relations déjà nouées.

De même, les réseaux sociaux sont perçus comme une solution à un autre problème politique saisi en ville, qui n'était d'ailleurs pas spécifique à Fontenay-sous-Bois : le manque d'intérêt des jeunes pour la politique. En effet, personne en dessous de 40 ans ne participe à une des réunions organisées dans le cadre des États Généraux de la Ville Numérique.

"On soubaitait toucher plus la population jeune car la démocratie locale n'est pas un sujet qui intéresse les jeunes, donc on a essayé de les attirer en faisant la réunion dans un bar un petit peu branché à Fontenay. Ca n'a pas du tout marché. C'était la réunion où il y avait le moins de monde, c'était dommage mais on a quand même réussi à avoir 2 personnes, la discussion, même si ce n'était pas ce à quoi on s'attendait, était intéressante ${ }^{13}$

Etant donné que les jeunes ${ }^{14}$ sont des utilisateurs avides des médias sociaux, on présente souvent les nouvelles technologies comme un des moyens possibles de les intéresser à la vie politique. Selon l'étude la plus récente de l'Observatoire des Réseaux Sociaux de IFOP ${ }^{15}$ l'année 2009 a été marquée en France par une amplification de l'essor des réseaux sociaux en ligne. 77\% des internautes interrogés déclarent appartenir à au moins un réseau social testé, et ce pourcentage atteint 96\% pour la tranche de 18-24 ans L'analyse des scores des réseaux par les sous-catégories d'âge montre que le score le plus élevé de chaque réseau est toujours atteint chez les jeunes et qu'il diminue avec l'âge des internautes. Ainsi, les lycéens et les étudiants sont les plus susceptibles d'avoir un compte Facebook (69\%). Les arguments soulignés par les adhérents de cette idée sont les suivants : premièrement, les jeunes auraient besoin de l'immédiateté et d'un échange rapide alors que les procédures démocratiques traditionnelles ne sont pas assez réactives. De plus, les jeunes seraient susceptibles de profiter de nouvelles formes de participation politique comme nous montre l'exemple des Canadiens cité par Amanda Clarke. Une étude sur la jeunesse canadienne réalisée en 2009 montre que 52\% des personnes interrogées ont créée un groupe sur Facebook ou ont adhéré à un tel groupe entre autres pour défendre une cause. $47 \%$ ont pris la parole à un

13 Entretien avec le webmestre du site dédié et le responsable du groupe.

14 A. CLARKE, Les médias sociaux. Utilisations politiques et conséquences pour la démocratie représentative. http://www2.parl.gc.ca/Content/LOP/ResearchPublications/2010-10-f.pdf, publié le 22/03/2010, consulté le $15 / 06 / 2010$

15 http://www.slideshare.net/azizhaddad/ifop-observatoire-rseaux-sociaux-janvier-2010 
débat en ligne et 39\% ont fait suivre un courrier électronique concernant des causes ${ }^{16}$ à défendre. Cependant, d'autres études montrent que certains jeunes se sentent harcelés quand les personnalités et les institutions publiques cherchent à adhérer à leurs réseaux sociaux.

Outre un facteur facilitant la communication politique, la présence sur les réseaux sociaux et la politique municipale en matière des TIC est également perçue comme un facteur de compétitivité de la ville par rapport aux autres. Dans cette perspective, il est important de noter que la municipalité fait partie du palmarès annuel organisé par l'association Villes Internet qui fait le classement des communes de toute taille qui mettent en ceuvre une politique significative en matière de démocratisation des $T I C$ et de leurs usages citoyens ${ }^{17}$. Depuis novembre 2009 la ville de Fontenay-sous-Bois est reconnue par l'association comme "Ville@@@@@ en se retrouvant ainsi parmi les meilleures du classement. Ce label, bien que n'apportant pas de profits matériels, contribue sans doute au prestige de la municipalité et augmente sa compétitivité par rapport aux autres. Le caractère volontariste de tous les investissements concernant les TIC est clairement mis en lumière dans la communication de la ville (à savoir le SIM et les Actes des États Généraux de la Ville Numérique). Les technologies d'information et de communication sont introduites dans les institutions diverses gérées par la municipalité telles que les écoles ou les Espaces Publiques Numériques. L'accent est également mis sur la promotion de l'Internet dans les quartiers défavorisés pour la réduction de la fracture numérique.

Ensuite la ville, comme une grande majorité de villes françaises, dispose d'un site internet municipal (SIM) http://www.fontenay-sous-bois.fr/. Le site est perçu comme "lieu de centralisation des informations» ${ }^{18}$ et comme un élément de la stratégie de diversification des outils de communication des collectivités. Aussi, le site municipal comprend des éléments d'interactivité. Il y a un forum intégré, plusieurs services administratifs sont disponibles en ligne, ainsi que la possibilité de contacter les élus.

Néanmoins, il faut souligner que ces nouveaux supports servent en réalité avant tout à la reproduction des informations existantes, comme par exemple le bulletin municipal et l'archivage d'anciens numéros disponibles en ligne. Par conséquent, le dispositif renouvelle les conditions d'accès à l'information mais pas son contenu. En effet, l'utilisation des TIC dans la communication n'est pas en rupture avec les procédures traditionnelles d'information. Au contraire, elle les complète.

16 A. CLARKE, op. cit.

17 http://www.villes-internet.net/UPLOAD/rubrique/pages/16/16_rubrique.php, consulté le 2 juillet 2010.

18 P. BOUQUILLION, I. PAILLIART, op. cit., p. 15. 


\section{Les enjeux de communication.}

La promotion des événements liés aux États Généraux de la Ville Numérique se révèle être un enjeu majeur d'un point de vue communicationnel. Les réseaux sociaux choisis ont été tous les deux exploités dans cette optique. C'est pourquoi, après avoir étudié les enjeux sociopolitiques de l'usage des réseaux sociaux par la mairie, il est indispensable de les analyser du point de vue communicationnel.

Premièrement, la fonctionnalité « événements » du compte Facebook est souvent utilisée. Quinze événements en tout ont été annoncés de cette façon. Le but originel est d'informer les membres du groupe des dates des réunions publiques. Néanmoins, ce qui était également important, c'était d'avoir un retour sur le niveau de participation des invités. Comme le rappelle dans l'entretien l'administrateur des comptes Facebook et Twitter du projet :

"On a aussi communiqué sur les réunions publiques. Et là on voit qu'il y a une réponse. Les gens disent je viens, je viens pas, je viens peut-être».

Bien que ce retour ne soit que déclaratif, il permet d'estimer le nombre de participants et de préparer la logistique. Cela permet aussi de prévenir un oubli éventuel de la part des participants. Nous pouvons souligner que les plus importants fonctionnaires de la mairie confirment toujours leur présence, comme pour attirer plus de public et de se montrer engagés dans le projet. C'est notamment le cas du maire qui a été présent seulement à la réunion du lancement des États Généraux et qui a pourtant déclaré venir à la majorité de réunions publiques de soir.

Deuxièmement, quant à Twitter, il est utilisé uniquement pour rappeler des réunions du soir organisées avec la participation du public. Le but était clair : créer un buzz et stimuler le bouche-à-oreille autour du projet. Cet usage du réseau social par les porteurs du projet va de soi au vu des fonctionnalités de Twitter ${ }^{19}$, comme le rappelle dans l'entretien l'administrateur du compte Twitter du projet :

"On a envoyé des messages sur Twitter. Là on a informé les gens des réunions publiques. Ce vecteur de communication a permis aux gens de venir, a rappelé la réunion aux gens qui avaient oublié. "

Les twitts, étant des messages courts, servaient très bien pour les annonces et les rappels des événements. Aussi, l'émetteur de ces messages peut être sûr de les envoyer uniquement aux personnes intéressées au projet (système des abonnés aux twitts) et ainsi les personnaliser selon les profils de ces abonnés.

L'usage des réseaux sociaux dans le but communicationnel repose aussi sur les liens hypertextes pour tenir l'internaute informé du projet et pour le mobiliser à prendre la parole dans la procédure de concertation. Ce qui saute aux yeux lorsque l'on analyse le contenu du compte Facebook des États Généraux de la Ville Numérique, c'est le nombre important des liens

19 http://blog.lefigaro.fr/hightech/2008/03/twitter-pour-les-nuls.html, publié le 7/03/2008, consulté le 15/06/2010. 
intégrés dans les postes publiés sur ce compte. Ils sont présents dans les billets publiés sur le wall, dans les annonces des événements, dans la présentation du compte. De prime abord le compte Facebook du projet est donc très ouvert et mène l'internaute vers d'autres sites web. Néanmoins, la majorité de ces liens hypertextes conduit en effet vers le site web dédié au projet. La mairie suit donc la stratégie des entreprises, qui gardent les internautes le plus longtemps possible dans leur réseau.

\begin{tabular}{|c|c|c|c|}
\hline Position & Origine & Visites & Pourcentage \\
\hline 1 & accès direct & 3515 & 57.34 \\
\hline 2 & moteurs de recherche & 1313 & 21.42 \\
\hline 3 & liens référents & 1302 & 21.24 \\
\hline Total & & & $\mathbf{1 0 0}$ \\
\hline
\end{tabular}

Tableau 1 - La répartition des internautes visitant le site dédié au projet selon la façon d'accéder au site

Paradoxalement également, la minorité de liens présents sur le compte ne menant pas vers le site web spécifique du projet font malgré tout se focaliser l'internaute sur ce même sujet. En effet, ces liens conduisent soit au site municipal de la ville de Fontenay, soit vers les sites partenaires du projet comme celui de l'association Villes Internet ou ARTESI.

Alors que dans le secteur économique, une telle fermeture du territoire du site a pour but de fidéliser l'internaute et de l'amener vers l'acte d'achat, dans le cas analysé il s'agit plutôt de le familiariser avec le projet politique et de le convaincre de s'y joindre. Aussi, cette stratégie permet de générer le trafic du site dédié au projet municipal, ce qui peut ensuite être utilisé par les porteurs du projet comme argument pour convaincre les décideurs politiques de l'intérêt que les habitants de la ville portent au projet. Les données provenant du backoffice du site dédié montrent que dans certains cas les liens ont apporté du trafic du site dédié. Comme le rapporte dans l'entretien l'administrateur du site :

"Seulement $38 \%$ passaient par la page d'accueil pour naviguer sur le site. Caa veut dire que le reste passait par un lien direct $"$.

Ce qui est encore plus frappant, c'est que certains visiteurs du site dédié provenaient des sites où la mairie n'a pas publié de liens de manière officielle ou volontaire. Ce même interviewé détaille dans l'entretien que :

"Il y a eu des entrées sur le site depuis www. linkedin.com ce qui est marrant car on n'a jamais communiqué sur ce réseau sur les États Généraux. Et pourtant il y avait des gens qui se passaient le lien ».

Cet exemple montre l'importance des réseaux sociaux aujourd'hui. Un ou plusieurs porteurs du projet ont probablement mis le lien vers le site événementiel, qui a ensuite été partagé par d'autres membres du réseau. Voilà 
pourquoi une stratégie efficace d'utilisation des réseaux sociaux consiste non seulement à la publication du plus grand nombre d'informations et de liens sur de multiples sites web, mais aussi au contrôle des informations publiées hors de la campagne institutionnelle.

Cependant, selon les données fournies par le webmestre du site événementiel, le trafic de ce site a été généré de manière minime par les réseaux sociaux exploités.

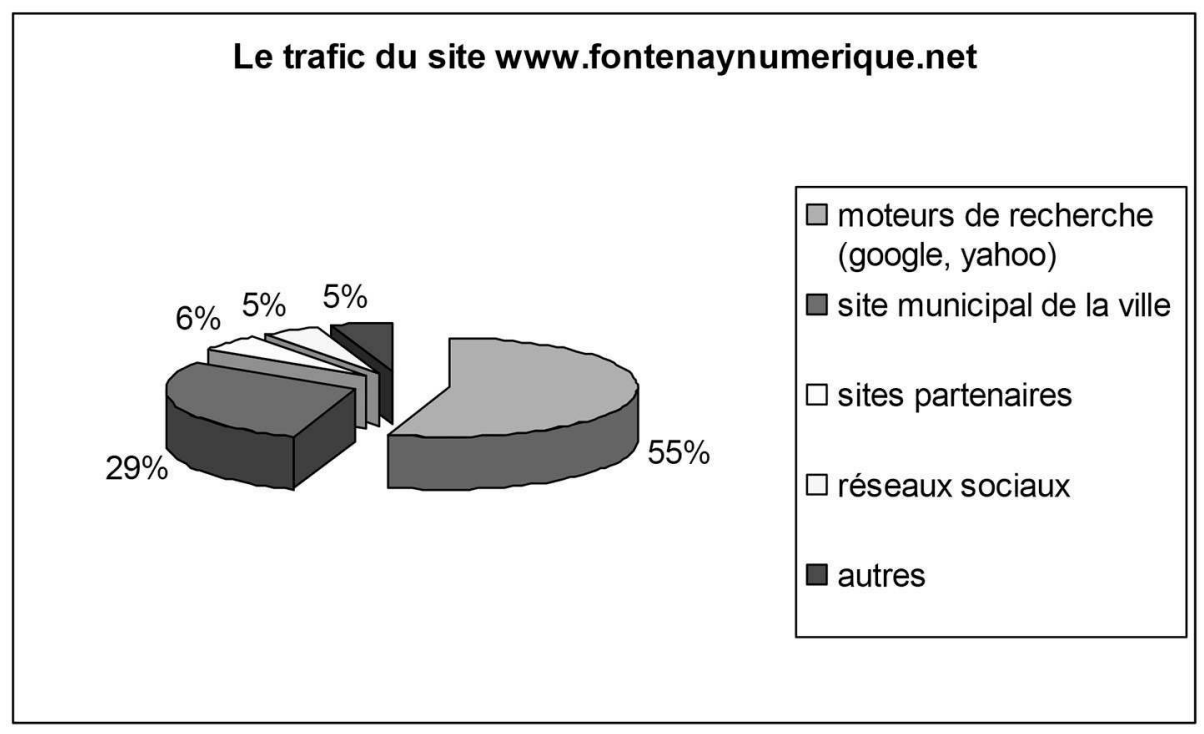

Figure $1^{20}$

Comme le montre le graphique, seulement $5 \%$ d'internautes qui venaient vers le site dédié d'un autre site y étaient amenés par les réseaux sociaux. En comparaison avec le taux de visites provenant des moteurs de recherche et du site municipal de la ville, il est clair que les attentes des porteurs du projet par rapport aux réseaux sociaux ne se sont pas confirmées.

\section{Quelles limites à cette conquête?}

L'objectif de cette dernière partie est de démontrer pourquoi il y a un tel décalage entre les attentes des porteurs du projet par rapport à la communication sur les réseaux sociaux et la réalité observée. Pourquoi est-ce que la présence des États Généraux sur Twitter et Facebook n’a pas apporté autant que souhaité ?

20 Ce graphique a été créé à la base des statistiques fournies par le webmestre du site dédié. Faute de son accord, nous ne sommes pas en mesure de publier ces statistiques en annexe. 


\section{Une participation quasi inexistante.}

Une première limite à l'efficacité des réseaux sociaux dans la communication sur le projet municipal est hétérogène aux porteurs du projet et provient des usagers des réseaux sociaux exploités.

La première difficulté rencontrée réside dans la faible participation des internautes. Le directeur de la DSIT avoue en entretien que :

"Il est difficile d'attirer les gens, de leur faire naviguer sur un site, surtout s'il demande une réflexion».

C'est pourquoi, pour augmenter le nombre de membres du compte Facebook du projet, les chargés du projet se sont appuyés sur les communautés virtuelles déjà existantes en mobilisant la page Facebook de Fontenay-sous-Bois ainsi que la page officielle Facebook du maire de la ville. Malgré cela les membres du groupe des États Généraux comme les abonnés au compte Twitter du projet n'étaient pas nombreux. Quant à Facebook, le nombre des membres s'élève à 176. Quant à Twitter, 22 internautes suivent les messages émis sur le compte des États Généraux. Afin d'estimer la valeur réelle de ces chiffres-là, il est indispensable de se référer à une norme. Je vais m'appuyer ici à la loi Metcalfe et à la loi Reed selon lesquelles la valeur d'un réseau social dépend fortement du nombre d'usagers de ce réseau ${ }^{21}$. Selon Metcalfe, la valeur d'un réseau est proportionnelle au carré du nombre de nœuds qui y sont connectés. Selon Reed, les réseaux qui encouragent la construction des groupes communicants créent une valeur qui croît de façon exponentielle avec la taille du réseau.

Certes, si le nombre de membres du réseau est pris en compte comme l'unique variable, la valeur de ces comptes doit être estimée comme très basse. Le nombre de membres du groupe Facebook des États Généraux ou le nombre d'abonnés aux twitts des porteurs du projet est visiblement inférieur à la moyenne observée sur Internet. À titre de comparaison, la page Facebook d'Issy-les-Moulineaux, une autre ville en banlieue parisienne attire plus de 2000 fans.

Néanmoins, ce calcul trop simpliste a ses limites. Une valeur égale est attribuée à chaque réseau social alors qu'ils ne sont pas utilisés avec la même intensité. Pour affiner l'analyse il faudrait donc en plus étudier les profils des membres du groupe Facebook du projet ainsi que l'intensité des échanges entre les nœuds du réseau.

L'analyse des profils des membres du groupe Facebook du projet apporte une conclusion importante. Une grande majorité d'entre eux travaille à la mairie ou participe activement à la vie publique locale et par conséquent entre en contact avec les agents de l'Hôtel de Ville. Parmi les membres du groupe Facebook on retrouve aussi des relatifs individus associés aux porteurs du projet. Toutefois, dans un souci de rigueur scientifique, il est important de mentionner que je n'étais pas en mesure d'analyser tous les profils des membres 
du groupe Facebook étudié à cause du nombre limité d'informations révélées par certains utilisateurs.

Pour analyser les données accessibles, nous allons nous appuyer sur la théorie des liens faibles et des liens forts de Mark Granovetter. Selon cette théorie, les liens forts sont ceux que l'on a entretenus avec les membres de sa famille, avec des amis proches et des collègues réguliers. Autrement dit, les liens forts naissent des rapports directs et de long terme, caractérisés par une réciprocité élevée. A contrario, les liens faibles sont noués à court terme lors de rapports très contextuels, ponctuels voire occasionnels ${ }^{22}$.

Etant donné qu'une grande majorité de membres du compte Facebook du projet travaillent ensemble à la mairie, leurs liens peuvent être qualifiés de liens forts. Paradoxalement, la force de ces liens diminue la valeur des réseaux analysés. Elle rend moindre la chance de disséminer les informations sur le projet vers des réseaux différents car les internautes appartiennent aux mêmes réseaux réels et virtuels. Aussi, comme les membres du groupe Facebook sont liés par les liens forts, ils se ressemblent. Cela peut faire du groupe Facebook un groupe trop homogène et empêcher une vraie discussion dans le sens d'un échange d'arguments opposés.

Non seulement les membres du groupe Facebook du projet liés entre eux par des liens forts ne sont pas nombreux, mais aussi le taux de participation à la discussion lancée par les gestionnaires de communauté est très faible. Cette observation est contradictoire au leitmotiv du discours des enthousiastes du web 2.0 qui prévoient un passage de la passivité de la consultation antérieure à une posture de participation beaucoup plus engagée de la part de l'internaute ${ }^{23}$.

En analysant le compte Facebook du projet, de prime abord on a l'impression que beaucoup de personnes participent au débat en postant des messages sur le wall. Néanmoins, après avoir repéré ces personnes on se rend vite compte que ce sont uniquement les administrateurs/animateurs du site qui contribuent. Les seules contributions des internautes viennent des représentants des associations qui essaient de nouer un partenariat et faire passer leurs intérêts ainsi que faire parler des événements auxquels ils participent.

Après avoir étudié les postes publiés sur le wall, il est clair que les porteurs $\mathrm{du}$ projet tentent en vain de faire discuter les internautes. Non seulement les membres du groupe Facebook du projet ne prennent pas l'initiative de lancer un sujet de débat, mais personne ne réagit non plus aux questions posées par les administrateurs du groupe.

Le débat entre les administrateurs du groupe Facebook et les internautes n'est pas le seul élément manquant. Au contraire, en analysant la page Facebook des États Généraux de la Ville Numérique, nous pouvons nous rendre compte du grand vide communicationnel qui y règne. Cet espace virtuel est privé de tout signe d'engagements de la part des utilisateurs.

22 M. GRANOVETTER, «The Strength Of Weak Ties ». American Journal of Sociology, 78, 1973, 1360-80. 23 F. REBILLARD, Le web 2.0 en perspective, L'Harmattan, Paris, 2007, p. 38. 
Le fait d'adhérer à la page Facebook du projet est le seul indicateur qui confirme une sorte de lien entre les internautes et la page du projet. Toutefois, il n'est pas suffisant pour qu'on puisse parler d'engagement. L'acte d'adhésion à un groupe sur Facebook est un acte très facile, instantané, gratuit et massif. Très souvent les utilisateurs adhèrent à plusieurs groupes auxquels ils ne se sentent pas pour autant attachés. De plus, dans le cas de la page du projet, beaucoup d'internautes y adhèrent par obligation car ils travaillent à la mairie. L'adhésion est pour eux une liaison suffisante, ils ne veulent pas aller plus loin et s'engager dans quoi que ce soit sur la page Facebook.

Aussi, même les manifestations moins exigeantes que la discussion comme par exemple le fait de cliquer sur le bouton «jaime ça »/ « je n'aime pas ça » ne sont pas choisies par les internautes. Dans le peu de commentaires qu'on retrouve sur le wall de la page il n'y a pas d'émoticônes. Enfin, les gens ne publient pas de photos ni d'images et ne commentent pas non plus celles déjà publiées.

\section{Les limites provenant des manques du gestionnaire de communauté.}

Outre les freins provenant de l'attitude des usagers, nous avons également pu observer quelques limites relatives à la stratégie de communication, plus particulièrement des manques liés à la gestion de communauté. Ces freins se manifestent dans la forme des messages publiés ainsi que dans la façon d'animer le groupe dédié aux États Généraux de la Ville Numérique. Tout d'abord, le contenu des comptes Facebook et Twitter du projet n'était pas toujours adapté aux dispositifs utilisés. Davantage d'anomalies peuvent être observées sur le compte Facebook. Quant à Twitter, le contenu est assez bien adapté à l'outil. Les messages «twittés » sont courts et ils annoncent des événements à venir. La longueur des messages est dictée par les limites formelles de Twitter (maximum de caractères : 140). Cet usage du compte Twitter du projet provient de l'habitude de se servir des téléphones mobiles et des SMS.

Quant à la page Facebook, l'usage est beaucoup plus compliqué et novateur. C'est un outil qui laisse aux administrateurs beaucoup de liberté quant à la taille et à la forme des postes. De prime abord, en regardant surtout la rubrique événements, le contenu semble adapté au médium. Les annonces des réunions publiques sont courtes et écrites de manière publicitaire. En revanche, après avoir cliqué sur l'événement pour en savoir plus, l'internaute accède à un message institutionnel traditionnel, très formel, qui ressemble à une lettre officielle envoyée par l'administration. Cela montre clairement que même s'ils utilisent les nouveaux médias, les agents municipaux restent attachés à la forme traditionnelle de communication ${ }^{24}$.

Aussi, l'alimentation des comptes Facebook/Twitter très irrégulière. Très souvent plusieurs billets sont publiés en même temps alors que par la suite

24 Etant donné la longueur du message analysé, nous avons décidé de ne pas l'insérer dans le corps du texte. En revanche, pour le consulter merci de vous référer à l'annexe. 
aucun autre message n'est posté pendant les semaines qui suivent. Cela n'encourage pas la fidélisation des internautes qui ne sont pas incités à revenir vers la page du projet pour suivre la discussion ou les informations. Les internautes peuvent aussi avoir l'impression que la page Facebook du projet n'intéresse pas tellement les administrateurs et que le projet en tant que tel n'est pas dynamique et n'évolue pas. Par conséquent, ils risquent de perdre leur intérêt pour le projet.

Enfin, la communication sur le projet via les réseaux sociaux reste toujours une communication verticale. Elle n'est pas différente de la communication institutionnelle de la mairie qu'on peut trouver sur le site municipal ou sur le site dédié au projet. Facebook devient donc une arène de plus sur laquelle les messages sont disséminés. Les community managers y publient des invitations aux réunions ainsi que les informations sur les réunions qui ont déjà eu lieu. On poste aussi des messages pour décrire le projet municipal de manière détaillée. Par contre, les administrateurs du groupe semblent avoir oublié que fournir des informations, ce n'est pas lancer un débat. Par conséquent, les internautes ne se sentent pas concernés et ne s'expriment pas sur le site perçu comme un canal institutionnel de plus.

Ensuite, les communautés virtuelles créées autour du compte Twitter et Facebook du projet ne sont pas alimentées ni gérées par une personne chargée de cette mission. Au contraire, les tâches d'un community manager sont accomplies par plusieurs personnes qui partagent cette fonction et qui s'en occupent hors de leur activité professionnelle quotidienne. Le webmestre du site événementiel qui est en même temps un des contributeurs aux comptes Facebook et Twitter dit dans un entretien :

"J'ai fait au mieux mais sans pouvoir vraiment creuser car la gestion du site et la communication sur le site c'était en plus de mon activité"».

Cette affirmation montre que le manque d'effectifs peut nuire à la communication via les réseaux sociaux. Les effectifs réduits ont pour conséquence une multiplication de community managers qui sont au nombre de quatre. Comme ils ont chacun son vision de la gestion de la communauté virtuelle, leur présence sur Facebook n'a pas cette fonction unificatrice souvent attribuée aux community managers. Il n'est pas non plus possible de retrouver dans l'alimentation du groupe Facebook du projet une stratégie partagée et cohérente.

De plus, une web cartographie locale n'a pas été identifiée. Les porteurs du projet n'ont pas exploité de blogs ou comptes Facebook liés à la Ville mais créés par des individus et des groupes extérieurs à la mairie (associations, habitants). Les partenariats sont très souvent utilisés par les administrateurs des sites web ou les community managers car ils assurent une publicité gratuite de l'événement ou de la marque.

Finalement, la faiblesse de la communication via les réseaux sociaux est aussi causée par un manque de coopération entre les porteurs du projet et 
les médias institutionnels de la ville. Dans l'entretien l'administrateur des comptes Facebook et Twitter avoue que :

"C'étaient surtout le site de la ville et les autres médias de la ville qui n'ont pas vraiment communiqué qu'il y avait un site des États Généraux. Résultat? Facebook, Twitter; très peu d'abonnés. Il faut dire qu'il n'y a pas de politique locale concernant les nouveaux médias sociaux. "

\section{Conclusion}

Dans notre article, la question du rôle des réseaux sociaux dans la promotion d'un projet municipal est analysée à partir de l'exemple concret de la communication numérique sur le projet Etats Généraux de la Ville Numérique. Cette démarche conduit à examiner dans quelle mesure les réseaux sociaux constituent à la fois un enjeu et un outil difficile à manipuler pour les collectivités locales.

Premièrement, étant donné que les enjeux des États Généraux de la Ville Numérique sont étroitement liés aux nouvelles technologies, l'exploitation des outils de la communication numérique pour promouvoir le projet semble aller de soi. Toutefois, contrairement à cette première impression, afin de comprendre l'usage des réseaux sociaux par les collectivités territoriales, il est important de le placer dans un contexte politique et communicationnel plus large. D'un côté, cette stratégie de communication peut résulter de l'ensemble d'actions de la municipalité dans le domaine des nouvelles technologies qui ont déjà sensibilisé les élus et l'administration aux fonctionnalités de ces nouveaux outils. De l'autre, elle peut correspondre à une tendance plus générale de l'usage de nouvelles technologies par les collectivités locales et constituer une ressource de la collectivité qui reste en compétition avec d'autres institutions publiques à l'échelle locale.

Deuxièmement, la présence des collectivités locales sur les réseaux sociaux présente de nombreux enjeux. Ils peuvent être d'ordre informationnel (tenir le public informé, rappeler les réunions etc.), politique (rapprocher les différents acteurs du territoire et rapprocher les habitants et les élus dans le cadre d'une concertation) ou bien communicationnel (augmenter le trafic du site dédié, créer un buzz, capter et retenir l'internaute).

Troisièmement, malgré l'identification de ces enjeux, les stratégies de communication publique à travers les réseaux sociaux ne sont pas toutes réussies. Faute de capital humain (pas de community manager), d'analyse préalable d'usages numériques des habitants et d'expérience (la présence sur les réseaux sociaux considérée comme une expérience), le public peut ne pas adhérer à cette forme de la communication publique.

L'usage des réseaux sociaux par les municipalités peut être appréhendé comme une forme de réponse des institutions locales à l'évolution de l'espace public médiatique dominant d'une part et à la " crise " de la 
démocratie représentative ${ }^{25}$ d'autre part. Comme le montre l'exemple de Fontenay-sous-Bois, elles y voient non seulement un enjeu de communication et de circulation d'informations mais aussi de mobilisation et de participation politique. Il est néanmoins essentiel de saisir à travers la recherche empirique la portée effective de ces arènes alternatives pour la communication politique la participation. Le cas analysé rappelle que les réseaux sociaux peuvent être perçus comme un catalyseur des pratiques politiques, mais n'en constituent pas la cause.

Nous sommes conscients que les résultats de cette analyse, portée sur un cas précis, ne sont pas généralisables ${ }^{26}$. Faute de recherches plus poussées, il est difficile de tirer des conclusions bien arrêtées sur le rôle que peuvent jouer les médias sociaux dans la promotion d'un projet municipal. Pour en juger, il faudrait étudier les cas d'autres municipalités, différentes en ce qui concerne entre autres la structure sociodémographique de la population ou les usages d'Internet sur le territoire. Néanmoins, cette étude ouvre une autre voie pour les recherches sur le rôle des réseaux sociaux pour les collectivités locales en complétant les analyses existantes portant sur un échantillon homogène mais qui ne permettent pas de saisir les spécificités des cas étudiés.

\section{BIBLIOGRAPHIE}

BARABEL M. et al., "Les médias sociaux au service du marketing territorial : une approche exploratoire », Management \& Avenir, 2/2010, n 32, p. 233-253.

BOUQUILLION P., MATHEWS J., Le web collaboratif. Mutations des industries de la culture et de la communication, Grenoble, Presses Universitaires de Grenoble, 2010.

BOUQUILLION P., PAILLIART I., Le déploiement des Tic dans les territoires, Grenoble, Presses Universitaires de Grenoble, 2006.

CLARKE A., Les médias sociaux. Utilisations politiques et conséquences pour la démocratie représentative, http://www2.parl.gc.ca/Content/LOP/ResearchPublications/2010-10-f. pdf, publié le 22/03/2010, consulté le 15/06/2010.

DUPUIS P., Twitter, nouvelle Arme de Communication Événementielle, http:// www.darkplanneur.com/2009/10/twitter-nouvelle-arme-de-communicationev\%C3\%A9nementielle.html, publié le 06/10/2009, consulté le 14/06/2010.

FARIS D., Dissent and Revolution in a Digital Age: Social Media, Blogging and Activism in Egypt, Londres, I.B.Tauris, 2012.

GRANJON F., « La réduction de la fracture numérique », B. MASQUET dir., La démocratie électronique, Paris, La Documentation française, 2007, p. 27-38.

25 Pour une synthèse de discours sur la crise de la démocratie représentative, lire Y. SINTOMER, Petite histoire de l'expérimentation démocratique : Tirage au sort et politique d'Athènes à nos jours, Paris, Editions La Découverte, 2011.

26 D'ailleurs, si l'on regarde d'autres villes où la stratégie de communication numérique est plus élaborée (par exemple Issy-les-Moulineaux présent sur Facebook et possédant son réseau social i-Folio), on constate que la dynamique de leur compte n'est pas meilleure que celle du compte des États Généraux. 
GRANOVETTER M., "The Strength OfWeak Ties », American Journal of Sociology, 78, 1973, p. 1360-1380.

GREFFET F., Continuerlalutte.com. Les partis politiques sur le web, Paris, Presses de Sciences Po « Académique », 2011.

LEFEBVRE A., Les réseaux sociaux. Pivot de l'Internet 2.0, MM2 Editions, Paris, 2005.

LOISEAU G., WOJCIK S., « La démocratie électronique municipale ou la captation contrariée », in Cochoy F. dir., La captation des publics, Toulouse, Presses Universitaires du Mirail, 2004, p. 209-235.

LOISEAU G., « Les sites internet des municipalités, les blogs et les réseaux sociaux: vers une multiplication des modalités participatives dans l'espace public local? " in E. FOREY et C. GESLOT dir., Internet, machines à voter et démocratie, Harmattan, Paris, 2011, p. 191-213.

PIGENEL R., Les réseaux sociaux de parti politique servent-ils à quelque chose?, http://www.variae.com/les-reseaux-sociaux-de-parti-politique-servent-ils-a-quelquechose $\%$ C2\%A0/, publié le 22/01/2010 et consulté le 16/04/2010.

PROULX S., Web social: mutations de la communication, Presses de l'Université du Québec, 2010 (rééd.).

REBILlARD F., Le Web 2.0 En Perspective - Une Analyse Socio-Économique De L'Internet, L'Harmattan, 2007.

ROUQUETTE S., L'analyse Des Sites Internet - Une Radiographie Du Cyberespace, De Boeck, 2009.

SINTOMER Y. ,Petite histoire de l'expérimentation démocratique: Tirage au sort et politique d'Athènes à nos jours, Paris, Editions La Découverte, 2011.

TALBOT D., « How Obama Really Did It », Technology Review, 111, 5, p. 78-83, 2008.

WESTLING M., "Expanding the Public Sphere : The Impact of Facebook on the Political Communication", Society, vol. 28, 2007, p. 835 - 860.

http://www.slideshare.net/azizhaddad/ifop-observatoire-rseaux-sociaux-janvier-2010

http://blog.lefigaro.fr/hightech/2008/03/twitter-pour-les-nuls.html, publié le 7/03/2008, consulté le 15/06/2010. 
Annexe 1 Message publié sur Facebook analysé dans le rapport

De prime abord la forme du message semble être très bien adopté au médium choisi : l'Internet. Les auteurs de l'invitation ont créé un message riche en moyens d'expression. Il y a donc une photo suggestive d'une fille qui tient la Terre dans ses mains et le sujet du message est formulé de manière publicitaire. Il est composé d'une question et d'une phrase impérative, ce qui donne au message un caractère dynamique. Aussi, grâce au formulaire fourni par le système informatique de Facebook que l'auteur de l'invitation doit remplir, les informations les plus importantes telles que la date et le lieu de la réunion, sont mises en avant.

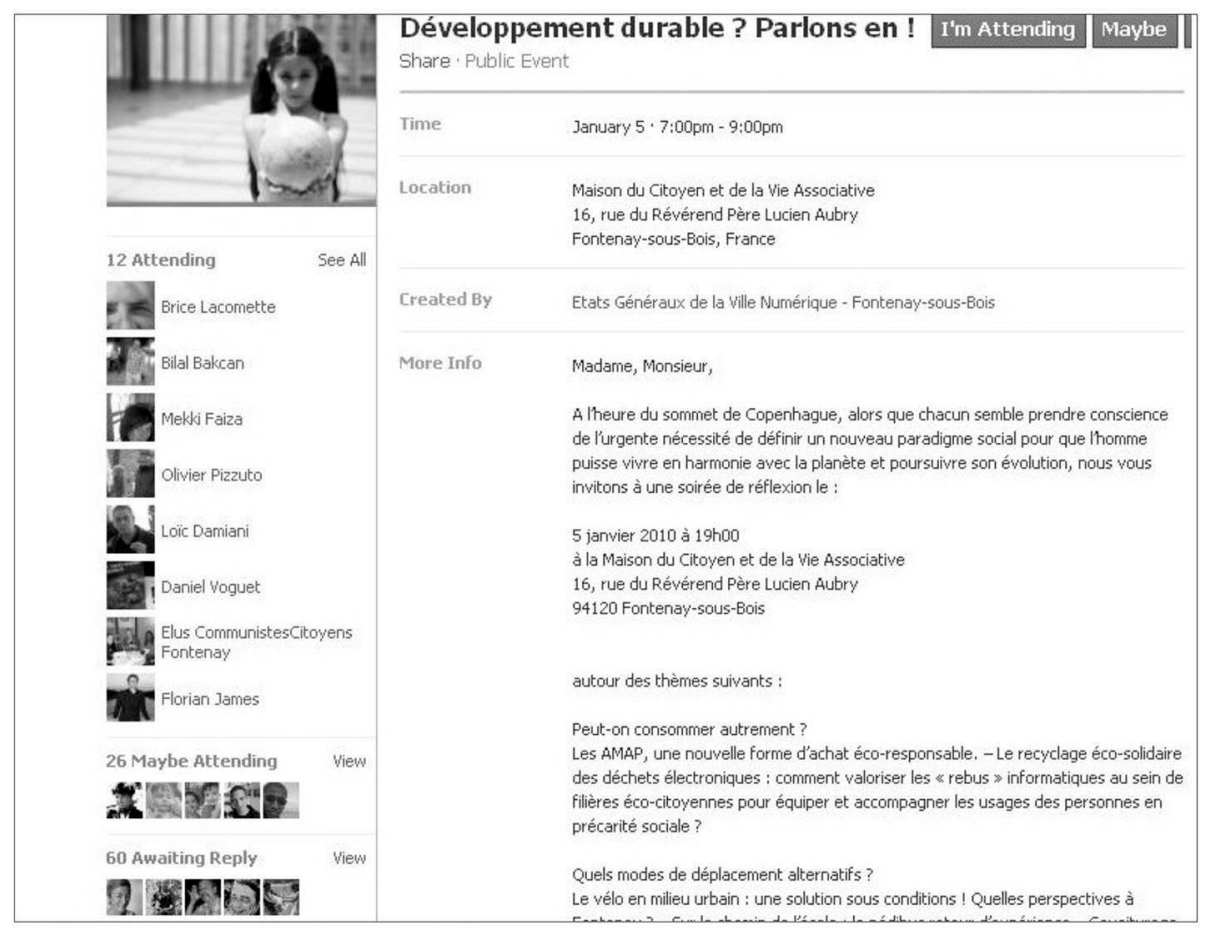

Figure 2 - Capture d'écran de l'invitation à la réunion sur le développement durable

Toutefois, quand l'internaute accède à la totalité du message, il voit un texte qui ressemble dans sa forme plus à une lettre institutionnelle qu'à un billet publié sur l'Internet. Tout d'abord, c'est un texte très long, beaucoup trop long pour l'internaute habitué à une lecture zapping. Aussi, sous le message nous retrouvons une signature très officielle du conseiller municipal qui organise la réunion. Cela construit une sorte de distance qui devrait être plutôt raccourci grâce au médium choisi. 


\section{Voici le texte de l'invitation:}

Madame, Monsieur,

À l'heure du sommet de Copenhague, alors que chacun semble prendre conscience de l'urgente nécessité de définir un nouveau paradigme social pour que l'homme puisse vivre en harmonie avec la planète et poursuivre son évolution, nous vous invitons à une soirée de réflexion le :

5 janvier 2010 à 19h00...à la Maison du Citoyen et de la Vie Associative 16, rue du Révérend Père Lucien Aubry 94120 Fontenay-sous-Bois autour des thèmes suivants :

Peut-on consommer autrement?

Les AMAP, une nouvelle forme d'achat éco-responsable. - Le recyclage écosolidaire des déchets électroniques : comment valoriser les « rebus » informatiques au sein de filières éco-citoyennes pour équiper et accompagner les usages des personnes en précarité sociale?

Quels modes de déplacement alternatifs?

Le vélo en milieu urbain : une solution sous conditions! Quelles perspectives à Fontenay? - Sur le chemin de l'école : le pédibus retour d'expérience - Covoiturage est-ce que ça marche?

Vers une économie solidaire?

Favoriser l'économie sociale pour un autre comportement économique : peut-on créer des pépinières d'entreprises issues de la filière éco-sociale - retour d'expérience.

Numérique et Développement Durable : Un paradoxe ou une opportunité ?

Présentation de la démarche des États Généraux de la Ville Numérique et des ateliers de réflexion qui se dérouleront de janvier en mars 2010.

Vous pouvez d'ores et déjà réagir poser des questions et formuler des propositions sur le site www.fontenaynumerique.net

Dans l'attente de vous rencontrer et de débattre avec vous, vous remerciant par avance de votre participation, nous vous prions de recevoir, Madame, Monsieur en l'expression de nos salutations les meilleures.

S’inscrire en ligne pour la réunion : http://www.fontenaynumerique.net/groupesde-travail/d\%C3\%A9veloppement-durable-1/

Conseiller municipal délégué à la Ville Numérique

Figure 3: texte de l'invitation analysée 
Résumé : Les municipalités françaises, qui misent de plus en plus sur la communication numérique, marquent depuis peu leur présence sur les réseaux sociaux. Ce constat provenant de l'observation de la toile est confirmé par certains chercheurs en marketing territorial et en sciences de l'information et de la communication. Pourtant, les recherches existantes se focalisent sur les conséquences de l'usage des réseaux sociaux par les municipalités tout en restant relativement silencieuses sur les motifs de ce choix et les raisons pour lesquelles elles consacrent du temps et de l'énergie à ces nouvelles arènes publiques, comme si l'usage des réseaux sociaux allait de soi. Notre contribution, appuyée sur l'étude de cas de la promotion d'un projet municipal sur les réseaux sociaux, vise à démontrer les enjeux de la présence des municipalités sur les réseaux sociaux. Nous distinguons les enjeux sociopolitiques comme l'objectif d'atteindre un public large et un public spécifique habituellement peu intéressé par le politique (les jeunes) ainsi que le but de devenir compétitif vis-à-vis d'autres municipalités. Les réseaux sociaux représentent également pour les municipalités des enjeux communicationnels comme la mobilisation des citoyens à travers un rappel des événements ou la création des hyperliens avec d'autres dispositifs web dédiés au projet. Cette perception du rôle des réseaux sociaux dans la promotion d'une politique publique est ensuite relativisée par une démonstration des limites de la stratégie de la forme de communication politique analysée.

Mots-clés : réseaux sociaux; communication politique ; municipalités ; communication numérique ; Facebook

Abstract : French municipalities have been increasingly present on social networks as confirmed
both by empirical examples and literature review. However, existing research remains relatively
silent on the reasons explaining this strategy, which in majority of cases is not very successful.
This chapter aims to demonstrate the challenges and the limits of the municipalities' presence on
social networks, studying a case of municipalproject promotion on Facebook and Twitter. I argue
that social networks represent diverse challenges for municipalities but their use is limited by the
lack of adapted strategy. Firstly, I distinguish the socio-political and communicational issues of
the phenomenon. Secondly, I identify the limits of this new form of political communication in
promoting public policy.

Keywords : Socialnetworks, Political communication, Municipalities, Digitalcommunication, Facebook, Twitter, Information, Participation 